\title{
Comprehensive bioinformatics analysis of the mRNA profile of PLCE1 knockdown in esophageal squamous cell carcinoma
}

\author{
XIAOBIN CUI ${ }^{*}$, HUAHUA XIN* ${ }^{*}$, HAO PENG and YUNZHAO CHEN \\ Department of Pathology and Key Laboratory for Xinjiang Endemic and Ethnic Diseases, \\ Shihezi University School of Medicine, Shihezi, Xinjiang 832002, P.R. China
}

Received January 13, 2017; Accepted July 17, 2017

DOI: $10.3892 / \mathrm{mmr} .2017 .7318$

\begin{abstract}
The authors previously reported that Phospholipase C epsilon 1 (PLCE1) exacerbated esophageal squamous cell carcinoma (ESCC), however, the underlying mechanism remains to be fully elucidated. The present study aimed to identify key differentially expressed genes (DEGs) and signaling pathways regulated by PLCE1 in ESCC. EC9706 and Eca109 cell lines were transfected with the specific small interfering (si) RNA of PLCE1, reverse transcription-quantitative polymerase chain reaction (RT-qPCR) and western blotting were performed to detect the expression levels of $P L C E 1$, and subsequently, mRNA array and multiple bioinformatics analysis were conducted. RT-qPCR was used to verify gene expression array results. The findings of the present study indicated that PLCE1 mRNA and protein expression were significantly suppressed $(\mathrm{P}<0.05)$ in the PLCE1 siRNA-transfected cells. In addition, a total of 223 DEGs with $>2$-fold alterations were screened between the PLCE1 siRNA-treated cells, including 168 upregulated and 53 downregulated DEGs. In particular, inflammation or immune-associated molecules, including Toll-like receptor (TLR)-4 interleukin-6, -8 and chemokine $\mathrm{C}-\mathrm{X}-\mathrm{C}$ motif ligand 2 were significantly increased following PLCE1 knockdown. Furthermore, Gene Ontology enrichment revealed terms associated with cell proliferation, differentiation, apoptosis, signal transduction, invasion and metastasis, which may potentially be associated with PLCE1 function. Kyoto Encyclopedia of Genes and Genomes pathway analysis demonstrated 46 pathways were disturbed by DEGs, including focal adhesion, mitogen activated protein kinase, TLR, p53 and janus kinase/signal transducer and activator of
\end{abstract}

Correspondence to: Dr Yunzhao Chen, Department of Pathology and Key Laboratory for Xinjiang Endemic and Ethnic Diseases, Shihezi University School of Medicine, North 4th Road, Shihezi, Xinjiang 832002, P.R. China

E-mail: cyz0515@sina.com

*Contributed equally

Key words: Phospholipase C epsilon 1 knockdown, esophageal squamous cell carcinoma, bioinformatics analysis transcription signaling pathways. The RT-qPCR results for validation of the selected DEGs were consistent with that of the microarray data. Overall, the results of the multiple bioinformatic analysis contributes to a systematic understanding of the roles of PLCE1 in ESCC.

\section{Introduction}

Esophageal squamous cell carcinoma (ESCC), which ranks the 6th in mortality and the 7th in morbidity in China, is one of the most prevalent malignant neoplasm around the world (1). Low intake of fruits and vegetables, poor nutritional status, smoking, and drinking all contribute to the etiology of ESCC (2). Despite improvements in ESCC treatments, the five-year survival rate of patients with ESCC is still low, because of poor understanding of the molecular pathogenesis of ESCC and infrequent early-stage diagnosis $(3,4)$. Although studies have indicated that some oncogenes and tumor suppressor genes are involved in the progression of esophageal cancer including Phospholipase C epsilon 1 (PLCE1), the molecular mechanisms are still poorly known. Therefore, it is imperative to study the functioning of genes that could be involved in the mechanism of the occurrence and development of ESCC.

Recently, a novel ESCC susceptibility loci located in chromosome10q23 in PLCE1 gene was identified by three genome-wide association studies (GWAS) in Chinese Han populations (5-7). Accumulated evidences have showed PLCE1 plays crucial roles in types of several cancers, such as head and neck (8), bladder (9-11), gastric $(12,13)$, skin (14), prostate (15). Our previous studies revealed increased of PLCE1 expression was significantly associated with lymph node metastasis and advanced TNM stage of Kazakh ESCC, which implicated that PLCE1 could be a novel biomarker for patients of Kazakh ESCC (16). Afterwards, we have stably knockdown PLCE1 by siRNA in ESCC cell lines Eca109 and EC9706, knockdown of PLCE1 resulted in an increase of the apoptosis, a decrease in cell proliferation as well as migration and invasion, and an inhibition of the formation of lamellipodia and filopodia of F-actin in vitro, the effect of PLCE1 on cell migration, invasiveness, and motility is correlated with epithelial-mesenchymal transition (EMT) and cytoskeleton dynamics (4). These results suggested that PLCE1 might play important roles in oncogenesis and progression of ESCC. 
Therefore, further research must be carried out to understand the exact role of PLCE1 in ESCC. To better understand the molecular mechanism of $P L C E 1$, the mRNA expression profile of PLCE1 knockdown was analyzed by the Affymetrix Gene Chip Human genome arrays. Nevertheless, a comprehensive analysis of the knockdown of PLCE1 has not been reported for microarray-based techniques in previous studies.

In this study, we explored the functional roles of PLCE1 on downstream genes and signaling pathways in ESCC. Hundreds of differentially expressed genes (DEGs), especially inflammation or immune-related genes, were identified in PLCE1-siRNA-treated cells compared with the control cells. Based on Gene Ontology enrichment and KEGG pathway analysis, we found that PLCE1 knockdown could impact a number of genes involved in proliferation, apoptosis, invasion and metastasis of tumor cells, et al and pathways including $M A P K$, Toll-like receptor, p53, Focal adhesion, et al. To the best of our knowledge, this is the first paper to perform a systematic analysis of how PLCE1 can influence DEGs and signal pathways in ESCC by microarray-based techniques.

\section{Materials and methods}

Cell culture and transfections. Esophageal cancer cell lines (Eca109 and EC9706) were purchased from Institute of Biochemistry and cell biology, Chinese Academy of Sciences (Shanghai, China) Cells was cultured in Dulbecco's modified Eagle's medium (DMEM) or RPMI-1640 (Gibco, Grand Island, NY, USA) containing $10 \%$ fetal bovine serum (FBS) at $37^{\circ} \mathrm{C}$ under $5 \% \mathrm{CO}_{2}$. The transfection of RNA was in the case of the final concentration of $5 \mathrm{nM}$ with HiPerFect transfection reagents (Qiagen, Hilden, Germany) in serum-free conditions. The target sequences for PLCE1-siRNA is 5'-AGCGUUGGU CCAUGCUUAATT-3'.

The DEGs. Raw data were normalized by MAS 3.0 algorithm. The main criterion for selection of DEGs between the two groups of samples being compared: i) q-value $\leq 5 \%$ (t-test); ii) fold change $\geq 2$ and $\leq 0.5$; given that there are three types of call values of each detected probe, at lowest one group among the values of three repetition should be non-A values. The expression level of the appropriate gene was the tallest number of $\mathrm{P}$ signs, for those genes including a lot of probes. A scatter plot was visualized to evaluate variations from chip to chip.

Gene ontology and pathway enrichment analyses. Molecular annotation system (MAS3.0) was used for gene ontology (GO) enrichment analysis. The 'conditional' option was designed to correct, while 'p value Cutoff' was designed to 0.05 . For those GO terms including at least one DEG, a false discovery rate (FDR) was performed by the Benjamini-Hochberg method. FDR was set to 0.1 (cutoff: 0.1).

Pathway enrichment analysis was used to perform via the Kyoto Encyclopedia of Genes and Genomes (KEGG; http://www.genome.jp/kegg/.html) database. The KEGG maps of biological functions associated with DEGs were identified. The P-value of the enrichment of DEGs in different pathways was reckoned by the super-geometric test, the significance threshold of false discovery rate (FDR) was also designed to 0.1.
Verification for gene expression using quantitative real-time polymerase chain reaction (qRT-PCR). Total RNA was extracted from ESCC cell lines with mRNA Extraction kit (Qiagen) according to the standard protocol. The cDNA of mRNA was synthesized with One Step PrimeScript mRNA cDNA Synthesis kit (Qiagen). All qRT-PCR data was standardized to $\beta$-actin expression. An ABI Prism 7500 Sequence Detection System (Applied Biosystems, Foster City, CA, USA) was used to detect the response. Amplification occurred at an initial denaturation cycle of $95^{\circ} \mathrm{C}$ for 5 min followed by 40 cycles of $95^{\circ} \mathrm{C}$ for $10 \mathrm{sec}, 60^{\circ} \mathrm{C}$ for $30 \mathrm{sec}$. All experiments were performed in triplicate at least three times. The $2-\Delta \Delta \mathrm{Cq}$ method was used to quantify the results. The primers used have been listed in Table I.

Western blot analysis. Transfected cells were lysed using RIPA buffer supplemented with Protease Inhibitor (PMSF). Protein was separated by SDS-PAGE, transferred to the PVDF membrane, and prevented by blocking with $0.1 \%$ Tween-20 in TBST including 5\% nonfat milk for $2 \mathrm{~h}$ at room temperature, then, membranes were incubated at $4^{\circ} \mathrm{C}$ overnight with primary antibodies against PLCE1 (1:200; rabbit. no. sc-368932; Santa Cruz Biotechnology, Inc., Santa Cruz, CA, USA) and $\beta$-Actin (1:500; rabbit. no. PR-0255; ZSGB-BIO, Beijing, China), and then incubated with secondary antibodies (1:10,000; rabbit. no. zf-0311; ZSGB-BIO) for $2 \mathrm{~h}$ at $37^{\circ} \mathrm{C}$. After washing, membranes were exposed in the darkroom. Finally, the objective bands were analyzed with the ECL procedure.

Statistics. Statistical analysis was carried out by Graphpad Prism 5.0e Software. Results were expressed as mean \pm standard deviation comparison between two groups. Differences between means were investigated by paired t-test within two groups. $\mathrm{P}<0.05$ was considered to indicate a statistically significant difference.

\section{Results}

Si-PLCE1 inhibited PLCE1 mRNA and protein expression. To determine the oncogenic function of PLCE1, si-PLCE1 was used to interfere with PLCE1 expression in Eca109 and EC9706 cells. The endogenous expression of PLCE1 was detected by qRT-PCR and western blot analysis after transfection. The expression of PLCE1 at both mRNA and protein levels was significantly reduced in the PLCE1 knockdown cells, compared with control groups (Fig. 1). These results indicated that the expression levels of PLCE1 mRNA and protein were markedly silenced after transfection in Eca109 and EC9706 cells.

Analysis of the DEGs. To explore the influence of PLCE1 on downstream genes, the mRNA expression profile was analyzed using the Affymetrix GeneChip Human genome 3, IVT arrays which contain 49294 genes in Eca109 and EC9706 transfected with PLCE1 siRNA compared to control groups.

Results compared with control groups, a total of 223 DEGs were identified by greater than 2.0-fold, of these, 168 DEGs were robustly upregulated and 55 DEGs were robustly downregulated. Primitively, the significantly increased genes were identified, such as DUSP5, IL8, IER3, IL6 and so on, while 
Table I. The primers used for Q-RT-PCR.

\begin{tabular}{|c|c|c|c|}
\hline Gene name & Primers & $\begin{array}{c}\text { Annealing } \\
\text { temperature }\left({ }^{\circ} \mathrm{C}\right)\end{array}$ & $\begin{array}{l}\text { The length of } \\
\text { product (bp) }\end{array}$ \\
\hline PLCE1 & $\begin{array}{l}\text { F:GAGCTGCAATCGAAGTCTGG } \\
\text { R:AAGGCCTTCTGTGAGTCCTC }\end{array}$ & 60 & 192 \\
\hline$I L-1 a$ & $\begin{array}{l}\text { F:ACGACTGGGTTTCAATCAGG } \\
\text { R:CTGCATGACTCGCCTTATCA }\end{array}$ & 60 & 142 \\
\hline$I L-1 b$ & $\begin{array}{l}\text { F:CCAGGATGAGGACCTGAGAA } \\
\text { R:CGAGGCATTTCTGTTGTTCA }\end{array}$ & 60 & 149 \\
\hline CXCl1 & $\begin{array}{l}\text { F:CCCAAACCGAAGTCATAGCC } \\
\text { R:GATTTGTCACTGTTCAGCATCTTT }\end{array}$ & 58 & 109 \\
\hline CXCL2 & $\begin{array}{l}\text { F:CGAAGTCATAGCCACACTCAAG } \\
\text { R:CTTCTGGTCAGTTGGATTTGC }\end{array}$ & 58 & 116 \\
\hline CCL2 & $\begin{array}{l}\text { F:GCACTCTCGCCTCCAGCATGA } \\
\text { R:CAGCAGGTGACTGGGGCATTGA }\end{array}$ & 50 & 121 \\
\hline CCL2O & $\begin{array}{l}\text { F:TGCTGTACCAAGAGTTTGCTC } \\
\text { R:CGCACACAGACAACTTTTTCTTT }\end{array}$ & 58 & 217 \\
\hline$\beta$-actin & $\begin{array}{l}\text { F:TGAAGTGTGACGTGGACATCCGC } \\
\text { R:GCCAATCTCATCTTGTTTTCTGCGC }\end{array}$ & 60 & 356 \\
\hline
\end{tabular}

F, forward; R, reverse.

A

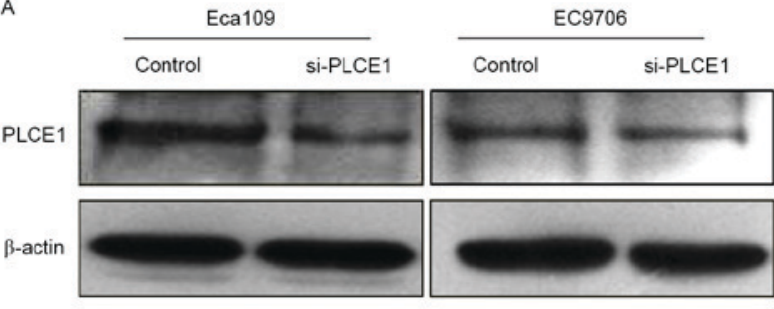

B

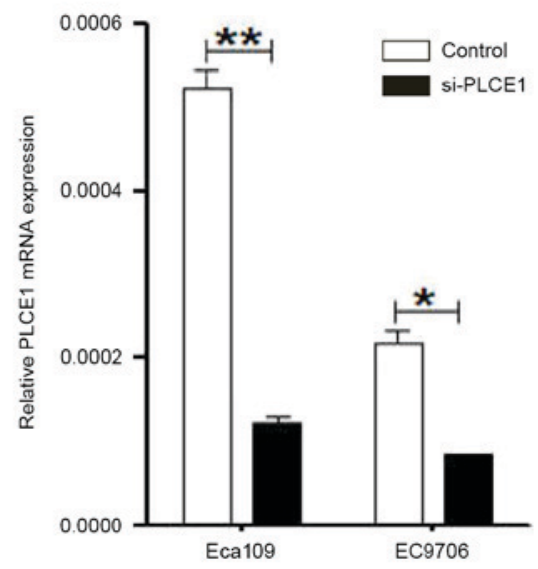

Figure 1. Effects of si- PLCE1 on the expression of PLCE1 in ECa109 and EC9706 cells. (A) PLCE1 mRNA expression was examined by qRT-PCR $\left({ }^{*} \mathrm{P}<0.05,{ }^{* *} \mathrm{P}<0.01\right){ }^{*} \mathrm{P}<0.05$. (B) Expression of PLCE1 protein was detected by western blotting. $\beta$-actin acted as the control.

significantly downregulated genes including TMEM30A, SNTB2, TMEM106B and so on (Table II). Interestingly, the expression of inflammatory factors was significantly increased after PLCE1 gene silencing, such as $I L-8, I L-6, C X C R 4$ and so on. Contrary to popular belief, inflammation was regarded as a promoter of tumor formation and metastasis. The results of

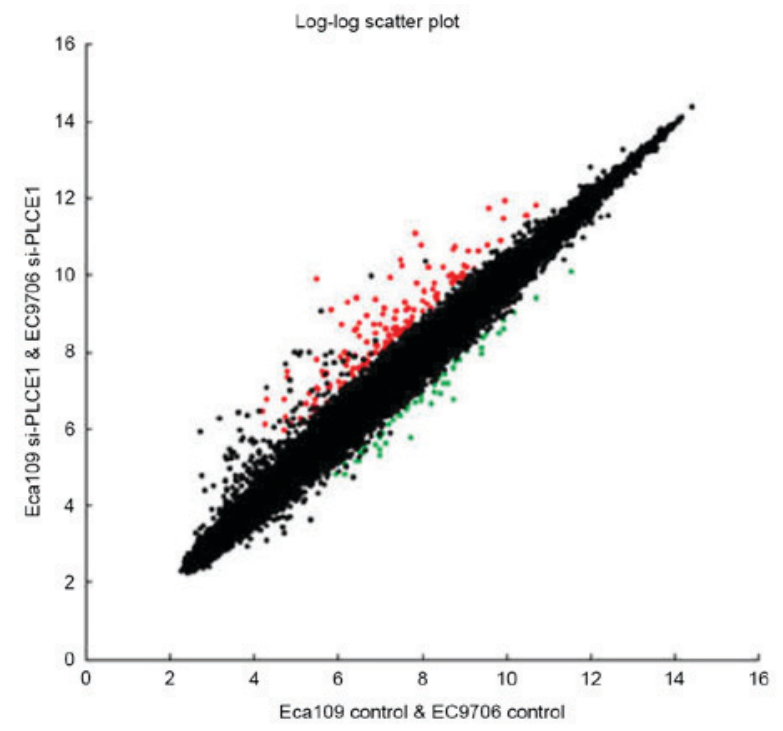

Figure 2. RNA array analyses of si-PLCE1-treated cells ECa109 and EC9706 cells compared to control cells. The log-log scatter-plot for the microarray results, red plots represent upregulated genes, while green plots represent downregulated genes.

our study suggested that PLCE1 might inhibit inflammatory factors expression. A two-dimensional rectangular coordinate plane was shown a scatter plot (Fig. 2). Collectively, PLCE1 might have a significant impact on expressions of PLCE1modulated downstream genes. Accordingly, a lot more researches are needed to figure out the possible relationship between these genes and PLCEI.

GO enrichment analyses. In order to have a command of the functional categories of that DEGs involved in and to find 
Table II. Differentially expressed genes in PLCE1 siRNA-Treated esophageal squamous cell carcinoma cells, compared with control cells.

A, Upregulated genes

\begin{tabular}{|c|c|c|c|c|c|}
\hline Gene symbol & Fold change & Gene symbol & Fold change & Gene symbol & Fold change \\
\hline IL8 & 22.624 & HSPA5 & 3.007 & TNRC6A & 2.27 \\
\hline CXCL2 & 10.224 & SAT1 & 3 & IFI16 & 2.26 \\
\hline$I F I 27$ & 10.111 & $C L D N 1$ & 2.966 & HSPA5 & 2.26 \\
\hline IFIT2 & 8.896 & PRIC285 & 2.929 & CXCR4 & 2.252 \\
\hline CXCL2 & 8.1833 & PLSCRI & 2.925 & SOD2 & 2.249 \\
\hline$O A S L$ & 7.9428 & PHLDA1 & 2.908 & $J U N$ & 2.246 \\
\hline 11762907_at & 7.457 & $R E L B$ & 2.908 & DUSP6 & 2.242 \\
\hline 11760093_at & 7.012 & $N F K B I Z$ & 2.879 & $F N D C 3 A$ & 2.241 \\
\hline IFIT1 & 6.821 & NT5E & 2.808 & $G D F 15$ & 2.238 \\
\hline DDIT3 & 6.788 & STC2 & 2.802 & SAMD9 & 2.232 \\
\hline$D D X 58$ & 6.5573 & OAS1 & 2.734 & HIF $1 A$ & 2.23 \\
\hline DDIT3 & 6.071 & $F N D C 3 A$ & 2.715 & $F N D C 3 A$ & 2.225 \\
\hline IFIT3 & 5.934 & SERPINE2 & 2.691 & TNRC6A & 2.214 \\
\hline IL6 & 5.899 & $F 3$ & 2.663 & ZC $3 H A V 1$ & 2.214 \\
\hline TRAC & 5.221 & ZC $3 H 12 C$ & 2.663 & IFITMI & 2.202 \\
\hline GDF 15 & 5.007 & SAT1 & 2.613 & PLSCR1 & 2.198 \\
\hline PTGS2 & 5.005 & $A D M$ & 2.61 & $A D R B 2$ & 2.197 \\
\hline ISG20 & 4.985 & $I R F 7$ & 2.596 & $C D K N 1 A$ & 2.194 \\
\hline TNFRSF 21 & 4.789 & $A B C D 3$ & 2.592 & SPINK1 & 2.193 \\
\hline IRF9 & 4.719 & OAS2 & 2.59 & $C L E C 2 B$ & 2.181 \\
\hline 11761908_at & 4.698 & $A D M$ & 2.562 & PMAIP1 & 2.157 \\
\hline$G D F 15$ & 4.418 & $F N D C 3 A$ & 2.522 & $\mathrm{ZC} 3 \mathrm{H} 12 \mathrm{C}$ & 2.156 \\
\hline PTGS2 & 4.31 & $I T G A 2$ & 2.506 & $F G F 2$ & 2.143 \\
\hline C5orf41 & 4.295 & IRF 1 & 2.503 & SIPAIL2 & 2.136 \\
\hline ISG15 & 4.122 & PARP9 & 2.503 & $F I C D$ & 2.129 \\
\hline$G D F 15$ & 4.091 & PLSCRI & 2.501 & DUSP6 & 2.114 \\
\hline TNFRSF 21 & 4.05 & DNAJB9 & 2.5 & HSPA5 & 2.112 \\
\hline$S T C 2$ & 4.024 & ICAMI & 2.495 & RSPO3 & 2.108 \\
\hline DUSP5 & 4.003 & $M A F F$ & 2.482 & ANGPTLA & 2.107 \\
\hline TNFAIP3 & 3.996 & FAIM3 & 2.475 & SAMHDI & 2.105 \\
\hline SERPINE2 & 3.81 & $S A A 1 / S A A 2$ & 2.47 & PPP1R15A & 2.103 \\
\hline PLSCR1 & 3.805 & STX11 & 2.454 & B3GNT5 & 2.086 \\
\hline RND3 & 3.796 & $C D K N 1 A$ & 2.441 & $F N D C 3 B$ & 2.078 \\
\hline TNFAIP3 & 3.743 & DUSP6 & 2.44 & USP18 & 2.076 \\
\hline AMIGO2 & 3.664 & ICAM1 & 2.427 & RND3 & 2.069 \\
\hline$P T G S 2$ & 3.533 & CXCR4 & 2.422 & $F N D C 3 B$ & 2.068 \\
\hline SERPINE2 & 3.492 & STAT1 & 2.415 & ELL2 & 2.062 \\
\hline HSPA5 & 3.483 & PMAIP1 & 2.407 & $J A G 1$ & 2.049 \\
\hline IFI6 & 3.462 & PTGER4 & 2.386 & MCL1 & 2.048 \\
\hline FOS & 3.427 & HERC5 & 2.385 & $T L R 4$ & 2.048 \\
\hline FOSL1 & 3.419 & FOSL1 & 2.378 & DRAMI & 2.044 \\
\hline HSPA5 & 3.417 & BIRC3 & 2.347 & OAS1 & 2.043 \\
\hline SAMD9L & 3.409 & BIRC3 & 2.346 & SIPAIL2 & 2.038 \\
\hline$A T F 3$ & 3.387 & $F G F 2$ & 2.346 & OTUD1 & 2.037 \\
\hline C5orf41 & 3.231 & PLSCR1 & 2.345 & GFPT1 & 2.032 \\
\hline FOSL1 & 3.196 & $V E G F C$ & 2.336 & $O S M R$ & 2.026 \\
\hline HIVEP2 & 3.148 & OAS1 & 2.335 & $G A D D 45 A$ & 2.023 \\
\hline BIRC3 & 3.131 & $J U N$ & 2.33 & ETV4 & 2.021 \\
\hline CLDN1 & 3.128 & HRASLS2 & 2.317 & PLAUR & 2.019 \\
\hline
\end{tabular}


Table II. Continued.

A, Upregulated genes

\begin{tabular}{lllll}
\hline Gene symbol & Fold change & Gene symbol & Fold change & Gene symbol \\
\hline CPA4 & 3.123 & ZFP36 & Fold change \\
FOSL1 & 3.12 & ABCD3 & 2.316 & JUN \\
IRAK2 & 3.089 & IFITM1 & 2.297 & IRF9 \\
IER3 & 3.081 & OAS1 & 2.294 & DUSP4 \\
GABBR1 & 3.052 & CXCR4 & 2.293 & SERPINE1 \\
RHEBL1 & 3.032 & SAMHD1 & 2.293 & KRT34 \\
SOD2 & 3.013 & DUSP6 & 2.281 & SLC6A6 \\
\hline
\end{tabular}

B, Downregulated genes

\begin{tabular}{|c|c|c|c|c|c|}
\hline Gene symbol & Fold change & Gene symbol & Fold change & Gene symbol & Fold change \\
\hline TMEMЗОА & 0.255 & C5orf53 & 0.418 & SEPPI & 0.468 \\
\hline TMEMЗОА & 0.259 & SUV39H1 & 0.42 & NEURLIB & 0.474 \\
\hline SNTB2 & 0.311 & NCRNA00201 & 0.426 & $M O B K L 2 B$ & 0.476 \\
\hline C21orf45 & 0.345 & GNA11 & 0.43 & LOXL1 & 0.477 \\
\hline IGSF8 & 0.348 & VEPHI & 0.439 & SUV39H1 & 0.478 \\
\hline STYX & 0.357 & $C A V 1$ & 0.446 & CHST13 & 0.48 \\
\hline C21orf45 & 0.36 & C8orf83 & 0.453 & PSKH1 & 0.481 \\
\hline STYX & 0.371 & $M O B K L 2 B$ & 0.454 & TMEM106В & 0.482 \\
\hline$D A Z A P 1$ & 0.374 & CLIC3 & 0.455 & ZNF362 & 0.483 \\
\hline C7orf41 & 0.385 & METTL7A & 0.456 & $B M P 8 B$ & 0.483 \\
\hline FAM107B & 0.398 & SORBS2 & 0.458 & SGOL2 & 0.486 \\
\hline CCNF & 0.399 & LMOI & 0.459 & TMEM106B & 0.486 \\
\hline FAM107B & 0.403 & $C B X 5$ & 0.46 & $F A M 47 E$ & 0.488 \\
\hline$H N R N P U$ & 0.404 & SORBS2 & 0.463 & $C B X 5$ & 0.49 \\
\hline SNTB2 & 0.406 & $C B X 5$ & 0.463 & $R N F 34$ & 0.491 \\
\hline GNA11 & 0.408 & SREK $1 I P 1$ & 0.464 & $A M Y 1 A$ & 0.492 \\
\hline$A L P I$ & 0.409 & SAMD11 & 0.465 & EXD3 & 0.494 \\
\hline \multirow[t]{2}{*}{ DPH3 } & 0.41 & $M X D 3$ & 0.468 & $P A B P C 4 L$ & 0.495 \\
\hline & & & & SUV39H1 & 0.497 \\
\hline
\end{tabular}

out which GO terms can be strongly enriched or significantly associated with our selected genes, GO term enrichment analysis was performed by MAS3.0. As is shown in Fig. 3, these DEGs were sorted and categorized into 26 different functional categories by PLCE1 knockdown in ECa109 and EC9706 cells. While 442, 178 and 94 significant GO terms for these DEGs were identified in Biological Process, Molecular Function and Cellular Component.

Remarkably, among the Biological Process, predominant terms were identified as follows. Firstly, the downregulated DEGs involved in cell division (GO:0051301), as well as upregulated DEG linked to cell cycle arrest (GO:0007050) and negative regulation of cell proliferation (GO:0008285) were represented, which revealed the inhibition of the PLCE1-mediated cell might block cell proliferation via these DEGs. Secondly, upregulated DEGs were enriched in anti-apoptosis (GO:0006916), negative regulation of apoptosis (GO:0043066), indicating theses terms might have direct or immediate adverse effect on apoptosis induction in PLCE1-siRNA-treated Eca109 and EC9706 cells. Thirdly, upregulated genes were also enriched in cell-cell signaling (GO:0007267), cell adhesion (GO:0007155), cell motility (GO:0006928), implying the terms might play a negative role in regulation of metastasis and invasion of PLCE1 knockdown cells. Fourthly, we also found that many DEGs were significant enrichment in regulation of transcription, DNA-dependent (GO:0006355), signal transduction (GO:0007165), G-protein coupled receptor protein signaling pathway (GO:0007186), cell surface receptor linked signal transduction (GO:0007166), which suggested these changes were likely to participate in the oncogenesis and tumor development of ESCC. To our interest, a significant proportion of clusters was enriched in 'response to virus (GO:0009615)', 'immune response (GO:0006955)', 'chemotaxis (GO:0006935)' and 'inflammatory response (GO:0006954)' in the biological process category, suggesting 


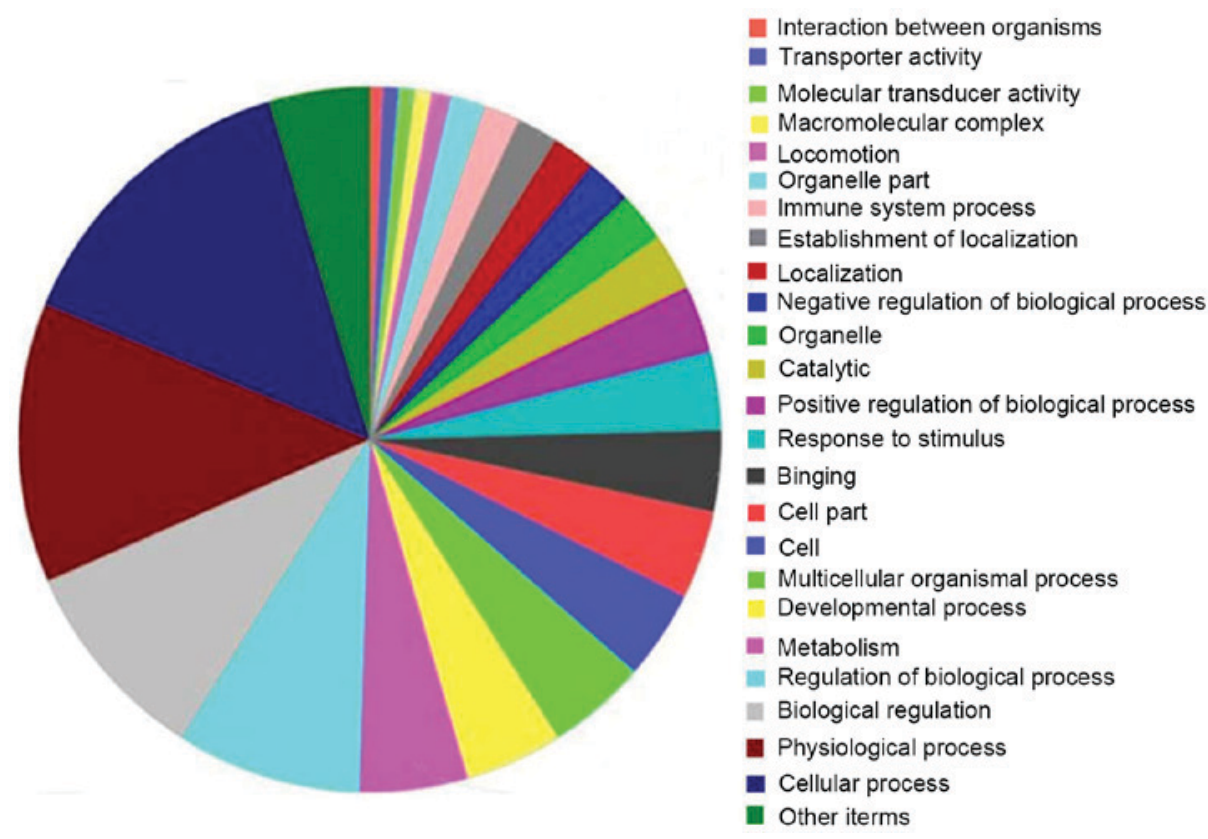

Figure 3. Functional classification of genes differentially expressed in PLCE1 knockdown cells. GO ontology software was used to analyze the list of differentially expressed genes classified according to biological process, molecular function and cellular component. These pie charts represent the distribution of the genes induced and repressed by $P L C E 1$ knockdown, pie sections are proportional to the number of genes.

that the changes related to these terms might take place during the genesis of ESCC.

Besides, a few terms connected to cytoskeleton organization were identified from Molecular Function and Cellular Component, including actin binding (GO:0003779), actin cytoskeleton (GO:0015629), focal adhesion (GO:0005925), integrin complex (GO:0008305), indicated that PLCE1 might exert significant influence in the metastasis, invasion and mobility of ESCC through the functions of genes implicated in these terms.

KEGG pathway enrichment analyses. To find out how the DEGs from PLCEl knockdown influence process of cell biological function, KEGG pathway enrichment analysis was carried out to identify relevant pathways. Based on the KEGG pathway analyses, these DEGs were enriched in 46 pathways which changed significantly (q-value $<0.05$ ), containing MAPK, Toll-like receptor, $p 53$, JAK-STAT signaling pathway, small cell lung cancer, renal cell carcinoma, bladder cancer, prostate cancer, cytokine-cytokine receptor interaction, focal adhesion, ECM-receptor interaction concluding consist of ITGA2, Starch and sucrose metabolism consisting of AMY1A, $A M Y 1 B, A M Y 1 B, A M Y 1 C, A M Y 2 A, A M Y 2 B, A M Y 2 B$, glutamate metabolism covering GFPT1, Nitrogen metabolism extending to NT5E and so on (Table III). The majority of these pathways were involved in cell biological function, including proliferation, invasiveness and apoptosis. Nevertheless, compared with the other signaling pathway, the majority of DEGs were significantly enriched in MAPK, JAK-STAT, P53, TLRs signaling pathways and the Focal adhesion. We should therefore concentrate our interest on the genes associated with the above pathways. The DEGs involved in the above signaling pathways are represented. There were alterations involved in the focal adhesion including 5 upregulated and 1 downregulated
DEGs. Among the MAPK signaling pathways -related genes, expression changes mainly occurred in 10 genes, including DUSP6, RELB, FGF2, FOS, DUSP4, DDIT3 (GADD153), $D U S P 5$, and $J U N$ between the PLCE1 siRNA and control group. The data of gene chip shown 4 upregulated genes (PMAIPI, SERAIPI (PAI-1), CDKN1A (P21), GADD45) in P53 signaling pathway, deregulated with a $>2$-fold difference following PLCE1 siRNA transfection in Eca109 and EC9706 cells. There were 7 upregulated genes (STATI, FOS, TLR4, $I L 6, I L-8)$ with $>2$-fold changes between the treatment and un-treatment cell involved in TLRs signaling pathways. Thus, our results indicated a strong interaction between PLCE1 and these pathways that DEGs were involved in.

Confirmation of genes associated with inflammatory factors by qRT-PCR after PLCE1-siRNA transfection. To approve or confute the results of our mRNA microarray analysis, qRT-PCR analysis was performed to evaluate mRNA levels of six pivotal DEGs selected in EC9706 and ECa109 transfected with PLCEI-siRNA. As expected, the results were consistent with the results of microarray findings that implicated involvement of $I L-1 a, I L-1 b, C X C L-1, C X C L-2, C C l-2$, $C C L-20$ genes. Compared with control group, by Q-RT-PCR analysis, all 6 genes were upregulated by PLCEl-siRNA treatment of EC9706 and ECa109 cells (Fig. 4). The results were corresponded to that of microarray data, which bore out the reliability of the results.

\section{Discussion}

ESCC, which is the fourth leading cause of cancer death, is one of the most aggressively malignant tumors in China (17). It was reported that $P L C E 1$ was a susceptibility gene inESCC.Although $P L C E 1$ has been extensively studied, it was not consistent with its 


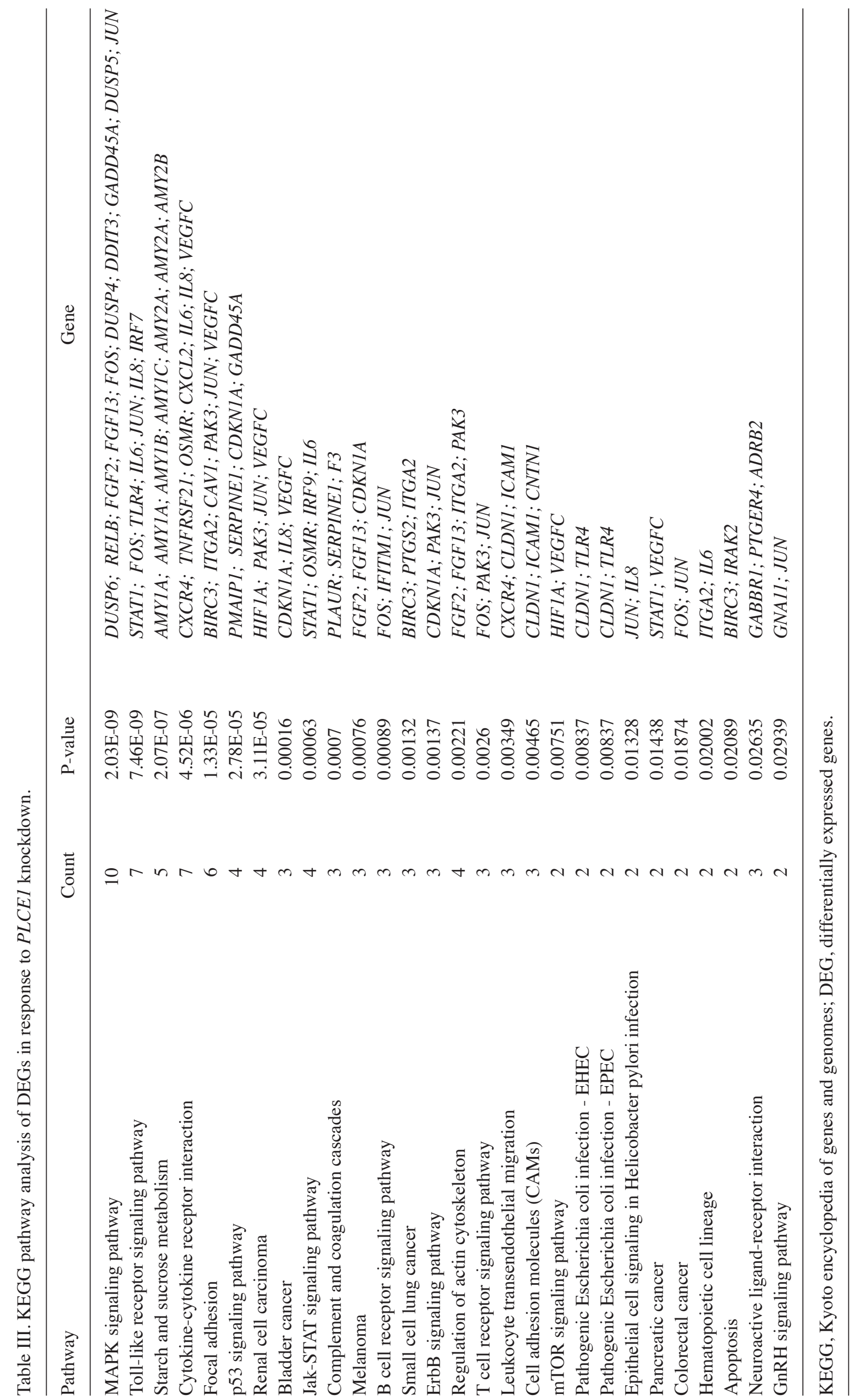



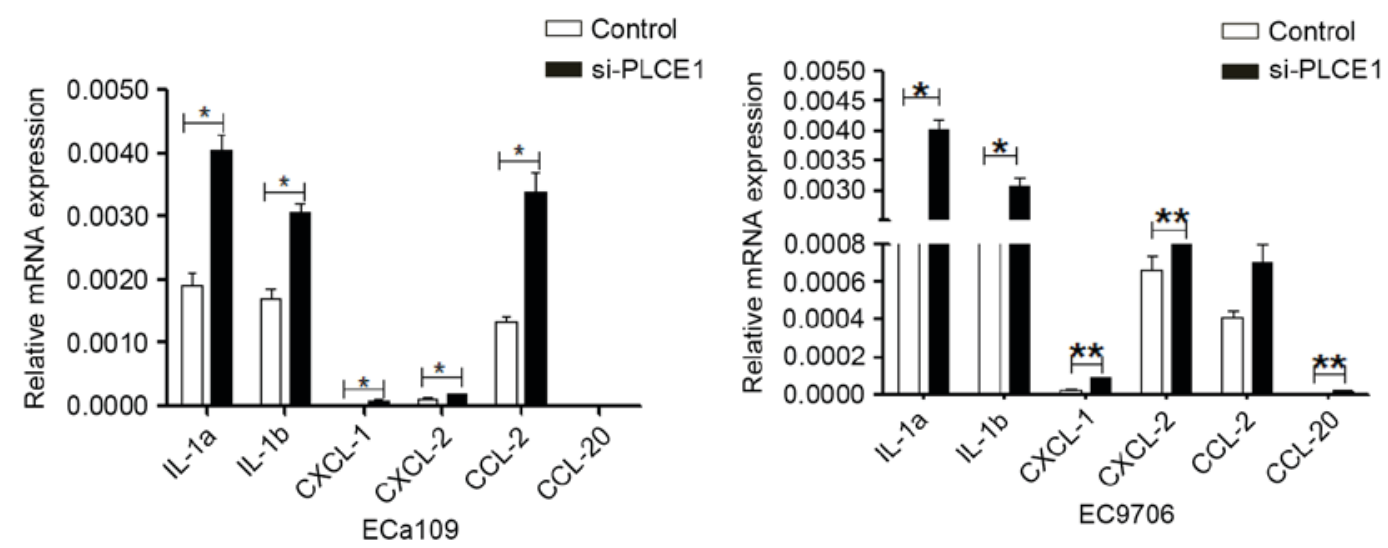

Figure 4. Q-RT-PCR validation for inflammatory factors. The relative expression of $I L-1 a, I L-1 b, C X C L-1, C X C L-2, C C L-20$ was significantly higher in PLCE1-siRNA-treated EC9706 and ECa109 cells than untreated control cell, respectively. ( $\left.\mathrm{P}<0.05,{ }^{* * *} \mathrm{P}<0.01\right)$.

role in cancer. Previous findings demonstrated PLCE1 has been identified as a tumor-suppressor gene of colorectal tumor (18). However, $P L C E 1$ has also acted as a novel oncogene in a variety of human tumors, such as bladder cancer (9) and head and neck cancer (8). Hence, PLCE1 promoting or inhibiting the occurrence and development of tumor is still to be further studied. Our previous studies showed increased expression of PLCE1 could be used as a potential biomarker for the early diagnosis and poor prognosis of ESCC (16). Furthermore, we also found $P L C E 1$ knockdown effectively inhibited cell growth/proliferation and increased apoptosis as well as reduced the invasion and migration in ESCC (4). Our results demonstrated that PLCE1 could play a tumor-oncogenic function in ESCC. Thus, it would be of great use to have systematic understanding of the functions of PLCE1. Here we presented a comprehensive analysis of mRNA profile of PLCE1 knockdown in ESCC cells by multiple bioinformatic analyses.

In the present study, a total of 223 DEGs, including 168 upregulated and 55 downregulated DEGs, were identified. In order to have a command of the functions of these DEGs, the DEGs were analyzed by GO enrichment tools. In the present study, these upregulated DEGs were significantly enriched in cell cycle arrest (PPPIR15A, DDIT3, CDKNIA, GADD45A, $I L 8)$, negative regulation of cell proliferation ( $P T G S 2, F G F 2$, IL6, CDKN1A, IFITM1, IL8), apoptosis (FGF2, CXCR4, TNFRSF21, PPP1R15A, RNF34, GADD45A, TNFAIP3, IER3, PHLDA1), cell-cell signaling (STC2, FGF2, ADM, GDF15, ISG15, IL8), cell adhesion (CLDN1, AMIGO2, RND3, ITGA2, $F 3$ ), these results supported such an assumption that oncogenic $P L C E 1$ was involved in esophageal proliferation, apoptosis and metastasis. Besides that, KEGG pathway enrichment analysis was carried out to identify relevant pathways, these DEGs were identified significantly enriched in 46 pathways. Among them, DEGs are closely linked to a variety of different signaling networks, including the MAPK, $p 53, J A K-S T A T$, Toll-like receptor signaling pathways. A following discussion focuses mainly on the alteration of genes involved primarily in $M A P K, p 53$, Toll-like receptor signaling pathways.

In the MAPK signaling pathway, several genes from the MAPK signaling pathway are altered in cells of PLCE1 downregulation, including 10 upregulated genes (DUSP6, DUSP4, DUSP5, RELB, FGF2, FGF13, FOS, DDIT3, GADD45A,
$J U N)$. Mitogen-activated protein kinase (MAPK) pathways, which has extracellular regulated kinases 1 and 2 (ERK1/2), c-Jun-N-terminal and p38, regulate many cellular functions including cell proliferation, differentiation, migration and apoptosis. Study showed that CDC25 homology domain of $P L C E$ exhibited GEF activity toward Rapl, transiting from the Rapl.GDP-bound state to the Rap1.GTP-bound state facilitated by GEF, leading to the accumulation of the formation of RaplGTP, thereby, which in turn stimulates B-Raf/MEK/ERK pathways within the cell, in addition, activation of Rap $2 B$ stimulates PLCE1, then activating H-Ras results in cascade response corresponding to $M A P K$, finally $M A P K$ was activated, and promoting cell proliferation. In addition, our previous study has shown that $E R K l$ protein expression was significantly increased in the ESCC tissues (19), thus it can be seen that PLCE1 might promote the incidence of esophageal carcinoma indirectly via MAPK/ERK1/2 pathways. Fibroblast growth factor 2 (FGF2) activated a series of intracellular signal transduction pathways by tyrosine kinase receptors $(F G F R)$ to form a complete pathway: FGF2/MAPK/ERK1/2 $(20,21)$, which finally induces various cellular activities, including mitosis, differentiation, proliferation, and cell migration $(22,23)$. However, DUSP6, DUSP5 and DUSP4, belonging to dual specificity phosphatase (DUSP) family, limits over-activation of the FGFs/MAPK/ERKI/2 signal transduction pathways in a negative feedback manner (24). This implies that the PLCE1 siRNA-induced apoptosis and proliferative inhibition in ESCC are related to DUSPS/FGFs/MAPK/ERKL//2 signal pathways. These findings provide a novel mentality to study the pathogenesis of ESCC in MAPK signaling pathway.

As known, $p 53$ is one of the most important tumor suppressor genes in cancer-associated diseases and plays a crucial role in regulating cell cycle arrest, apoptosis and senescence (25), recent study also revealed $p 53$ is involved in PLCE1-knockdown induced apoptosis in Esophageal Cancer cells (26). Our results demonstrated 2 key upregulated components CDKN1A (P21) and GADD $45 A$ involved in the $p 53$ signaling pathway were upregulated in PLCE1 siRNA treated cells. Following the downstream target of $p 53, p 21$ and GADD45A are activated and block the cell cycle at the G1 and G2 phases $(27,28)$. This result suggests that $P L C E 1$ inhibition may lead to an increase in p53 function by upregulating CDKN1A (P21) and GADD45A, 
and that a new $P L C E 1 / P 53 / P 21$ pathway is involved in $P L C E 1$-knockdown induced apoptosis and cell cycle arrest in ESCC. In addition, cellular senescence is primarily regulated by $p 53 / p 21$ and $p 16 / p R b$ pathways, where the $p 53 / p 21$ pathway mediates the replicative senescence and plays crucial roles in DNA damage response (29). Therefore, PLCE1 suppression might have an effect on the $p 53 / p 21$ signaling pathway through $p 53$ and $p 21$ upregulation, leading to proliferation inhibition and inducing cell senescence and in Eca109 and EC9706 cells.

In this study, we have shown that inflammation or immune-related molecules (TLR4, IL-8, IL-6, CXCL2) were significantly increased after PLCE1 suppression, and the results by qRT-PCR analysis were in agreement with that of microarray data. However, these results did not agree with recent reports in which $P L C E$ was shown to enhance tumor development in many human cancers (30-32), by increasing the production of inflammatory cytokines in local tissues, obviously, our results suggested that PLCE1 might be involved a yet unknown signaling pathway linking tumor promotion and cytokines. Interestingly, it was reported that the expression of cytokines $I L-1 a, I L-6$, and $I L-8$ initiated and supported cellular senescence (33). Similar report also showed that $I L-1 a$ activated tumor-suppressive pathways to maintain senescent growth (34). Nevertheless, cellular senescence restricts unlimited cell proliferation and plays an essential role in tumor suppression. Therefore, we investigate whether PLCE1 could enhance carcinogenesis and progression of ESCC via loss of cytokines-supported senescence. In addition, it was well recognized that toll-like receptor 4 (TLR4), an intensively studied member in the TLR family, results in transcriptional activation of pro-inflammatory genes including cytokines ( $I L-1, I L-6, C X C L-2)$ (35). Importantly, TLR4 activation inhibited hepatocellular carcinogenesis due to immune networks ( $I L-1 a / b, I L-6, C X C L-2)$ triggering cellular senescence (36). These results taken together suggested PLCEI could decrease cellular senescence via inhibition of the TLR4 signaling pathway, which contributed to the development of ESCC. In future studies, it will be worthwhile to determine whether PLCE1 has a role in esophageal carcinoma by loss of TLR4-mediated immunity supporting senescence.

In summary, the comprehensive understanding the role of PLCE1 in ESCC was obtained by bioinformatics workflow of mRNA profile after PLCE1 knockdown by multiple bioinformatics analysis. A total of 223 DEGs, 168 DEGs upregulated and 55 downregulated DEGs, were identified in PLCE1-siRNA-treated cells compared with the control cells. Alterations genes in the Focal adhesion, MAPK, P53 and TLRs signaling pathways may be important during PLCE1 siRNA-induced apoptosis, and proliferation, invasive and migrant inhibition of Eca109 and EC9706 cells. These findings provide information useful for combination targeted therapy in PLCE1-relevant neoplasm. We need to characterize DEGs and identify the exact molecular mechanism PLCE1 involved in proliferation, apoptosis, invasion and metastasis.

\section{Acknowledgements}

This study was supported by Grants from the National Natural Science Foundation of China (nos. 81560399, 81360358 and
81460362), the Major Science and Technology Projects of Shihezi University (no. gxjs2014 zdgg06), the Applied Basic Research Projects of Xinjiang Production and Construction Corps (no. 2016AG020), the high level talent project of Shihezi University (no. RCZX201533), and the Foundation for Distinguished Young Scholars of Shihezi University (no. 2015ZRKXJQ02).

\section{References}

1. Liu M, Hu Y, Zhang MF, Luo KJ, Xie XY, Wen J, Fu JH and Yang H: MMP1 promotes tumor growth and metastasis in esophageal squamous cell carcinoma. Cancer Lett 377: 97-104, 2016.

2. Wu M, Liu AM, Kampman E, Zhang ZF, Van't Veer P, Wu DL, Wang PH, Yang J, Qin Y, Mu LN, et al: Green tea drinking, high tea temperature and esophageal cancer in high- and low-risk areas of Jiangsu Province, China: A population-based case-control study. Int J Cancer 124: 1907-1913, 2009.

3. Cui XB, Zhang SM, Xu YX, Dang HW, Liu CX, Wang LH, Yang L, Hu JM, Liang WH, Jiang JF, et al: PFN2, a novel marker of unfavorable prognosis, is a potential therapeutic target involved in esophageal squamous cell carcinoma. J Transl Med 14: 137, 2016.

4. Cui XB, Li S, Li TT, Peng H, Jin TT, Zhang SM, Liu CX, Yang L, Shen YY, Li SG, et al: Targeting oncogenic PLCE1 by miR-145 impairs tumor proliferation and metastasis of esophageal squamous cell carcinoma. Oncotarget 7: 1777-1795, 2016.

5. Abnet CC, Freedman ND, Hu N, Wang Z, Yu K, Shu XO Yuan JM, Zheng W, Dawsey SM, Dong LM, et al: A shared susceptibility locus in PLCE1 at 10q23 for gastric adenocarcinoma and esophageal squamous cell carcinoma. Nat Genet 42: 764-767, 2010.

6. Wang LD, Zhou FY, Li XM, Sun LD, Song X, Jin Y, Li JM, Kong GQ, Qi H, Cui J, et al: Genome-wide association study of esophageal squamous cell carcinoma in Chinese subjects identifies susceptibility loci at PLCE1 and C20orf54. Nat Genet 42: 759-763, 2010.

7. Wu C, Hu Z, He Z, Jia W, Wang F, Zhou Y, Liu Z, Zhan Q, Liu Y, Yu D, et al: Genome-wide association study identifies three new susceptibility loci for esophageal squamous-cell carcinoma in Chinese populations. Nat Genet 43: 679-684, 2011.

8. Ma H, Wang LE, Liu Z, Sturgis EM and Wei Q: Association between novel PLCE1 variants identified in published esophageal cancer genome-wide association studies and risk of squamous cell carcinoma of the head and neck. BMC Cancer 11: 258, 2011.

9. Ou L, Guo Y, Luo C, Wu X, Zhao Y and Cai X: RNA interference suppressing PLCE1 gene expression decreases invasive power of human bladder cancer T24 cell line. Cancer Genet Cytogenet 200: 110-119, 2010.

10. Ling Y, Chunli L, Xiaohou W and Qiaoling Z: Involvement of the PLCe/PKC $\alpha$ pathway in human BIU-87 bladder cancer cell proliferation. Cell Biol Int 35: 1031-1036, 2011.

11. Zhang Y, Yan L, Zhao Y, Ou L, Wu X and Luo C: Knockdown of phospholipase C-epsilon by short-hairpin RNA-mediated gene silencing induces apoptosis in human bladder cancer cell lines. Cancer Biother Radiopharm 28: 233-239, 2013.

12. Xue W, Zhu M, Wang Y, He J and Zheng L: Association between PLCE1 rs2274223 A > G polymorphism and cancer risk: Proof from a meta-analysis. Sci Rep 5: 7986, 2015.

13. Yuan J, Li Y, Tian T, Li N, Zhu Y, Zou J, Gao J and Shen L: Risk prediction for early-onset gastric carcinoma: A case-control study of polygenic gastric cancer in Han Chinese with hereditary background. Oncotarget 7: 33608-33615, 2016.

14. Oka M, Edamatsu H, Kunisada M, Hu L, Takenaka N, Dien S, Sakaguchi M, Kitazawa R, Norose K, Kataoka T and Nishigori C: Enhancement of ultraviolet B-induced skin tumor development in phospholipase $\mathrm{C} \varepsilon$-knockout mice is associated with decreased cell death. Carcinogenesis 31: 1897-1902, 2010.

15. Wang Y, Wu X, Ou L, Yang X, Wang X, Tang M, Chen E and Luo C: PLCe knockdown inhibits prostate cancer cell proliferation via suppression of Notch signalling and nuclear translocation of the androgen receptor. Cancer Lett 362: 61-69, 2015.

16. Chen YZ, Cui XB, Hu JM, Zhang WJ, Li SG, Yang L, Shen XH Liu CX, Pan QF, Yu SY, et al: Overexpression of PLCE1 in Kazakh esophageal squamous cell carcinoma: Implications in cancer metastasis and aggressiveness. APMIS 121: 908-918, 2013. 
17. Cui XB, Pang XL, Li S, Jin J, Hu JM, Yang L, Liu CX, Li L, Wen SJ, Liang WH, et al: Elevated expression patterns and tight correlation of the PLCE1 and NF- $\kappa \mathrm{B}$ signaling in Kazakh patients with esophageal carcinoma. Med Oncol 31: 791, 2014.

18. Wang X, Zhou C, Qiu G, Yang Y, Yan D, Xing T, Fan J, Tang H and Peng Z: Phospholipase $\mathrm{C}$ epsilon plays a suppressive role in incidence of colorectal cancer. Med Oncol 29: 1051-1058, 2012.

19. Cui X, Li S, Li T, Pang X, Zhang S, Jin J, Hu J, Liu C, Yang L, Peng H, et al: Significance of elevated ERK expression and its positive correlation with EGFR in Kazakh patients with esophageal squamous cell carcinoma. Int J Clin Exp Pathol 7 : 2382-2391, 2014.

20. Akl MR, Nagpal P, Ayoub NM, Tai B, Prabhu SA, Capac CM, Gliksman M, Goy A and Suh KS: Molecular and clinical significance of fibroblast growth factor 2 (FGF2/bFGF) in malignancies of solid and hematological cancers for personalized therapies. Oncotarget 7: 44735-44762, 2016.

21. Pierozan P, Biasibetti H, Schmitz F, Ávila H, Parisi MM, Barbe-Tuana F, Wyse AT and Pessoa-Pureur R: Quinolinic acid neurotoxicity: Differential roles of astrocytes and microglia via FGF-2-mediated signaling in redox-linked cytoskeletal changes. Biochim Biophys Acta 1863: 3001-3014, 2016.

22. He Q, Ren X, Chen J, Li Y, Tang X, Wen X, Yang X, Zhang J, Wang Y, Ma J and Liu N: miR-16 targets fibroblast growth factor 2 to inhibit NPC cell proliferation and invasion via PI3K/AKT and MAPK signaling pathways. Oncotarget 7: 3047-3058, 2016.

23. Lee MN, Kim JW, Oh SH, Jeong BC, Hwang YC and Koh JT: FGF2 stimulates COUP-TFII expression via the MEK1/2 pathway to inhibit osteoblast differentiation in $\mathrm{C} 3 \mathrm{H} 10 \mathrm{~T} 1 / 2$ cells. PLoS One 11: e0159234, 2016

24. Rios P, Nunes-Xavier CE, Tabernero L, Köhn M and Pulido R: Dual-specificity phosphatases as molecular targets for inhibition in human disease. Antioxid Redox Signal 20: 2251-2273, 2014.

25. Majumder M, House R, Palanisamy N, Qie S, Day TA, Neskey D, Diehl JA and Palanisamy V: Correction: RNA-binding protein FXR1 regulates 21 and TERC RNA to bypass p53-mediated cellular senescence in OSCC. PLoS Genet 12: e1006411, 2016.

26. Li Y, An J, Huang S, Liao H, Weng Y, Cai S and Zhang J: PLCE1 suppresses p53 expression in esophageal cancer cells. Cancer Invest 32: 236-240, 2014

27. Tront JS, Huang Y, Fornace AJ Jr, Hoffman B and Liebermann DA: Gadd45a functions as a promoter or suppressor of breast cancer dependent on the oncogenic stress. Cancer Res 70: 9671-9681, 2010.
28. Cho JG, Park S, Lim CH, Kim HS, Song SY, Roh TY, Sung JH, Suh W, Ham SJ, Lim KH and Park SG: ZNF224, Kruppel like zinc finger protein, induces cell growth and apoptosis-resistance by down-regulation of p21 and p53 via miR-663a. Oncotarget 7: 31177-31190, 2016

29. Park H, Kim CH, Jeong JH, Park M and Kim KS: GDF15 contributes to radiation-induced senescence through the ROS-mediated p16 pathway in human endothelial cells. Oncotarget 7: 9634-9644, 2016.

30. Li M, Edamatsu H, Kitazawa R, Kitazawa S and Kataoka T: Phospholipase Cepsilon promotes intestinal tumorigenesis of Apc(Min/+) mice through augmentation of inflammation and angiogenesis. Carcinogenesis 30: 1424-1432, 2009.

31. Yang X, Ou L, Tang M, Wang Y, Wang X, Chen E, Diao J, Wu X and Luo C: Knockdown of PLCE inhibits inflammatory cytokine release via STAT3 phosphorylation in human bladder cancer cells. Tumour Biol 36: 9723-9732, 2015.

32. Oka M, Edamatsu H, Kunisada M, Hu L, Takenaka N, Sakaguchi M, Kataoka T and Nishigori C: Phospholipase Ce has a crucial role in ultraviolet B-induced neutrophil-associated skin inflammation by regulating the expression of CXCL1/KC. Lab Invest 91: 711-718, 2011

33. Oubaha M, Miloudi K, Dejda A, Guber V, Mawambo G, Germain MA, Bourdel G, Popovic N, Rezende FA, Kaufman RJ, et al: Senescence-associated secretory phenotype contributes to pathological angiogenesis in retinopathy. Sci Transl Med 8: 362ra144, 2016.

34. Freund A, Orjalo AV, Desprez PY and Campisi J: Inflammatory networks during cellular senescence: Causes and consequences. Trends Mol Med 16: 238-246, 2010.

35. Lin H, Yan J, Wang Z, Hua F, Yu J, Sun W, Li K, Liu H, Yang H, Lv Q, et al: Loss of immunity-supported senescence enhances susceptibility to hepatocellular carcinogenesis and progression in Toll-like receptor 2-deficient mice. Hepatology 57: 171-182, 2013.

36. Wang Z, Yan J, Lin H, Hua F, Wang X, Liu H, Lv X, Yu J, Mi S, Wang J and Hu ZW: Toll-like receptor 4 activity protects against hepatocellular tumorigenesis and progression by regulating expression of DNA repair protein Ku70 in mice. Hepatology 57: 1869-1881, 2013. 